\title{
Modelling the dietary impact of health-related claims on food labels in the UK
}

\section{Abstract}

Health-related claims (HRCs) are statements found on food packets that convey the nutritional quality of a food (nutrition claims) and/or its impact on a health outcome (health claims). Foods carrying HRCs have a slightly improved nutritional profile than foods without HRCs, however, it's unclear whether this translates into dietary improvements. We conducted a modelling study to measure the effect of HRCs on diet. As HRCs are already present on foods it is assumed that any impact that they have upon diet are already in effect. We modelled the impact on food purchases of removing HRCs, by assuming that the sales boost they receive is neutralised. These results can be inverted to estimate the current dietary impact of HRCs. Using the Living Costs Food (LCF) survey data, we calculate the average purchases and nutrient intake per person, per day. The LCF data is divided into sales of products with HRCs and sales of products without HRCs through solving mathematical equations combining LCF sales data with odds ratios from a meta-analysis examining the impact of HRCs on choices and data from a survey of foods examining the prevalence of HRCs and the nutritional quality of foods that carry them so that the sum of the sales of products with HRCs and without HRCs is equal to the total sales of products. Similarly, mathematical equations are solved that combine nutritional composition data with the sales of foods carrying and not carrying HRCs. In the baseline scenario foods carrying HRCs made-up $37 \%$ of the total purchases, and contributed $29 \%(559 \mathrm{kcal})$ of the total kcals purchased $(1907 \mathrm{kcal})$. When HRCs are removed from foods there is an average increase of $18 \mathrm{kcal} / \mathrm{d}(95 \%$ Uncertainty Intervals [UI] $-15,52),+2 \mathrm{~g} / \mathrm{d}$ increase in total fat $(95 \%$ UI $-1,4)$ and saturated fat $(95 \%$ UI $1,3)$, smaller changes are seen for protein $(+0.5 \mathrm{~g} / \mathrm{d}, 95 \% \mathrm{UI}-1,2)$, total sugar $(+0.5 \mathrm{~g} / \mathrm{d}, 95 \% \mathrm{UI}-4,7)$ and carbohydrate $(-0.5 \mathrm{~g} / \mathrm{d}$, $95 \%$ UI $-5,7)$. There is reduction in the amount of fruit $(-11 \mathrm{~g} / \mathrm{d}, 95 \%$ UI $-34,26)$ but an increase in vegetables $(+6 \mathrm{~g} / \mathrm{d}, 95 \%$ UI $-6,19)$. These results should be interpreted with caution due to the large uncertainty intervals. When HRCs are removed, we see a small deterioration in the quality of the average diet. If we invert these findings we can assume HRCs currently have a positive, albeit small, impact on diet.

\section{Funding information}

AK was funded by the European Commission 7th EU Framework Programme Small Collaborative Project CLYMBOL (contract no. 311963). PS and MR are funded by the British Heart Foundation (grant numbers: 006/PSS/CORE/2016/OXFORD and FS/15/34/ 31656). PS is also supported by the NIHR Oxford Biomedical Research Centre.

\section{Conflict of Interest}

There is no conflict of interest. 